chapter does not seem logically quite satisfactory. The radar method of determining distance is used on the basis of the velocity of light being constant. To some extent this is giving too much away, because the constant nature of the velocity of light arises from the conventional nature of distant simultaneity. This would not matter if it were not that the thing which is really difficult to understand about special relativity is how the velocity can be constant for different reference frames. The radar method when fully worked out, taking into account the conventional nature of time at distant points, does explain this. Chapter five deals with some consequences of the Lorentz transformation although the infamous clock paradox is moved to the special chapter seven at the end of the book. Chapter six is in many ways the least satisfactory part of the book. The author evidently does not wish to leave out of consideration general relativity, but within the technical bounds of what he can do, he is able to say very little about it. As a consequence, chapter six has an extremely cotton-wool texture. By the time one has reached the end of it, one feels very little wiser than when one began.

The book is nicely produced and extremely suitable for its purpose. Each chapter concludes with a number of numerical problems and the answers to these are given at the back of the book. The index is quite satisfactory.

C. W. Kinmister

\section{MASTERFUL PAPERS}

\section{Collected Papers of G. H. Hardy}

Including Joint Papers with J. E. Littlewood and others, Vol. 3. Edited by a committee appointed by the London Mathematical Society. Pp. 748. (Clarendon Press: Oxford; Oxford University Press: London, May 1969.) $105 s$.

EARLIER volumes in this handsome series dealt with Hardy's work (including collaboration) on number theory and the present volume is concerned with work on trigonometric series and the mean values of power series. The transition is a natural one, for the subjects are closely related. The work of Hardy and Littlewood in number theory led them to develop new methods of dealing with problems relating to summability of series and more than half of this book is taken up with papers on trigonometric series which are concerned with summability and related questions.

The theory of Fourier series is one of the central subjects of analysis and it has been responsible for many of the major developments in mathematics during the past two hundred years. The notion of function grew out of problems in the theory of convergence of Fourier series and both Riemann's and Lebesgue's definitions of the integral first appeared in the context of trigonometric series. Cantor's work in set theory was inspired by it and much of modern function theory and abstract harmonic analysis has close associations with the classical theory of Fourier integrals. Hardy and Littlewood enriched the whole theory, as the papers in this book amply demonstrate. It is impossible to describe all the aspects of their work in a short notice, but the following examples seem to me to be particularly elegant.

The first two groups of papers are concerned with convergence and summability questions. In the latter case, Hardy and Littlewood were concerned primarily with the problem of obtaining conditions for the Cesarro summability $(C, \delta)$ of the Fourier series at a given point for some $\delta \geq-1$. (No solution is known if $\delta$ is given in advance.) They obtained the complete solution: some Cesaro mean of the Fourier series of $f$ converges if and only if some Cesàro mean of $f$ converges.

The third section contains papers on the Young-Hausdorff inequalities, while the fourth and fifth are concerned with special series and a wide range of miscellaneous questions. One of the most remarkable results is the theorem of Hardy that the property

$$
\int_{0}^{1} t J_{v}\left(\lambda_{m} t\right) J_{v}\left(\lambda_{n} t\right) \mathrm{d} t=0
$$

is characteristic of the Bcssel function $J_{v}(t)$ of order $v$ and zeros $\lambda_{n}, \lambda_{n}$. (That is, the functions $\sin t$ and $t^{\frac{1}{2}} J_{y}(t)$ are the only function orthogonal with respect to their own zeros in $(0,1)$.)

The final section deals with mean values of power series. The chief problem here is the study of the mean value

$$
\mu_{p}(f ; p)=\frac{1}{2 \pi} \int_{-\pi}^{\pi}\left|f\left(\rho \mathrm{e}^{i \theta}\right)\right|^{p} \mathrm{~d} \theta
$$

of a function $f$ regular in the unit disk. The class of functions for which $\mu_{p}(f ; \rho)$ is bounded is the Hardy class $H^{p}$, which is of fundamental importance in the study of the Fourier series of functions in $L^{p}$. Hardy and Littlewood made outstanding contributions to this theory, a theory which has been extensively generalized to abstract spaces in recent years.

As in the earlier volumes, one cannot but be impressed by the power and beauty of the ideas here presented and of the elegance with which they are exposed. It is a pleasure to read these papers, which are not only of historical interest but which are also essential background material for a subject which is still of intense interest, albeit of extreme difficulty. Hardy and Littlewood's interest in the subject grew out of their work in number theory and it is entirely appropriate that the next major advances in the theory of trigonometric series probably await further developments in number theory.

\section{J. V. ARmitage}

\section{Correspondence}

\section{"Deka-deci" Notation}

SiR,-Whereas it is important for scientific and technical purposes to have a numerical notation which is both compact and unambiguous, each of the two forms of notation used at present has definite defects, particularly where large numbers or small (fractional) numbers are concerned; this is illustrated in Table 1. The first of these methods, the explicit method (column 1), is simple to apply, but with the quite common examples used here it is cumbersome and can easily lead to errors, while with a large number involving a sequence of zeroes (as in the example) there is no way of indicating how many of the zeroes represent significant figures rather than simply filling up the spaces in front of the decimal point. The second, index, method (column 2) is moro compact and it does enable the number of significant figures to be indicated; however, the index, being a superscript symbol, is visually and typographically inconvenient, and also being a small symbol it is easily disfigured if the text is produced or reproduced imperfectly.

Table 1. APPLICATION OF THE TWO COMMONLY USED FORMS OF NOTATION, AND OK THE PROPOSED "DEKA-DECI" NOTATION, TO A LARGE NUMBER AND A SMALI (FRACTIONAL) NUMBER*
"DAR

$12,300,000 \quad 1.23 \times 10^{7} \quad 1 \cdot 23 D 7$ ("One point two three deka seven") $0 \cdot 000,456 \quad 4.56 \times 10^{-4} \quad 4.56 d 4$ ("Four point five six deci four")

* Other slightly different forms of notation, for example, the European continental one with a comma in place of a full stop for the decimal point and so on, have been disregarded for the present purposes.

The application of the proposed "deka-deci" notation is demonstrated in the last column of Table 1; this method 
leads to a form even more compact than the index method, and avoids the disadvantages of the latter while retaining its useful features. One point to be noted is that, to standardize the method and also to give the most rapid indication of the order of magnitude of the figure involved, the number after the indicator letter ( $D$ or $d$ ), representing the power of ten involved, should preferably be chosen so that the number before the letter lies between one and ten, as in the examples given. One further point is that the indicator letter will always occur sandwiched between two numerals, except in the representation of pure powers of ten where it would often be permissible to represent, for example, ten million by $D 7$ rather than $1 D 7$; in cither case, however, because of its placing there should be no cause for confusion between this lotter and any mathematical symbols in the text or any abbreviations for units of quantities involved.

In certain instances it is possible to avoid altogether having to denote a large number or a small fraction by using metric prefixes to represent decimal multiples or submultiples of the units involved (the terms used here, "deka" and "deci", stem of course from the corresponding metric prefixes); this, however, is not always a convenient procedure if we are dealing with a series of figures ranging over several powers of ten, while it is not possible to do this at all if the quantities involved are pure numbers. In any case, the metric prefixes form a colloction to which it is absurd to apply the name "system"; the consistent adoption of the presently proposed notation would remove the need for such prefixes (except, perhaps, the most convenient ones between "milli-" and "kilo-") because it provides a much clearer and more direct indication of the order of magnitude involved, while this will also avoid the increasingly embarrassing problem of coining metric prefixes for multiples and submultiples outside the present limits.

Yours faithfully,

\section{P. Molyneux}

Department of Pharmacy,

Chelsea College of Science and Technology,

Manresa Road,

London SW3.

\section{Appointments}

Dr W. H. Gutt has been appointed head of the Building Research Station Materials Division; Dr L. H. Everett has been appointed deputy head.

\section{Announcements}

Professor G. A. Barnard, University of Essex, has been elected president of the Institute of Mathematics and its Applications.

The Science Research Gouncil invites applications from suitably qualified men and women engineers and teachers of engineering, for the 1970 Whitworth Foundation Awards. Candidates are asked to submit a paper of not more than 2,000 words, describing the project they would undertake if successful, by January 31,1970 . The awards may vary in length from three months to two years. Further details ean be obtained from the Science Research Council, State House, High Holborn, London WC1.

An international training course on Cell-Mediated Immune Reactions, with Special Emphasis on Molecular Aspects of Gell Surfaces will be held in Stockholm, June 8-18, 1970. The course will be sponsored by FMBO and will be organized jointly by members of the departments of immunology, tumour biology and bacteriology at the University of Stockholm and the Karolinska Institute Medical School. Details of application can be obtained from Professor P. Perlmann, Wenner-Gren Institute, Norrtullsgatan 16, S 11345 Stockholm, Sweden.
Awards under the Royal Society and Nuffield Foundation Commonwealth Bursaries Scheme have been made to the following: Professor T. C. Chambers (University of Melbourne) for study of the taxonomy, structure, evolution and geographical distribution of the fern genus Blechnum; Dr P. M. Clifford (McMaster University, Ontario) to study rock deformation; Dr F. R. Hewgill (University of Western Australia) for work on electron spin resonance techniques in studying oxidation of organic compounds; Professor P. R. Jefferies (University of Western Australia) to study methods used for research on microbiological oxidation of terpenoids; Dr G. A. R. Johnston (John Curtin School of Medical Research, Canberra) to study the active transport of likely amino-acid synaptic transmitters in the mammalian central nervous system; Dr K. V. Krishna Rao (Osmania University, Hyderabad) for research on organic and inorganic crystal structure analysis; Dr C. F. Lim (Univorsity of Singapore) to study the venomous marine snails of the family Conidae in the Australian area; Dr J. V. Lovett (University of New England, New South Wales) to work on cereals; Dr K. G. Marathe (University of Poona) to study modern methods in tracer applications and photochemistry; Dr Soon Ng (University of Malaya) to study various aspects of nuclear magnetic resonance spectroscopy; Dr A. M. Nhonoli (University College, Dar es Salaam) to learn new techniques in the investigation of anaemias; Dr R. L. Oliver (University of Adelaide) for work on granulite facies metamorphic rocks; Dr G. I. Opat (University of Melbourne) for research in theoretical elementary particle physics; Dr G. S. C. Pillai (Central Marine Fisheries Institute of South India) to study coral collections; Dr E. Senogles (University College of Townsville, Australia) to study gas phase free radical reactions; and Professor T. G. Vallance (University of Sydney) to develop and complete studies of altered mafic volcanic rocks and their metamorphic derivations from various areas in the British Isles.

\section{International Meetings}

March 4, Electroluminescent Solid State Devices, London (Meetings Officer, The Institute of Physics and the Physical Society, 47 Belgrave Square, London SW1). March 5-6, Relaxation Phenomena and Mechanical Properties of Polyvinylchloride, Strasbourg (Secretariat IUPAC, Macromolecular Division Conference on PVC, Centre de Recherches sur les Macromolecules, 6 rue Boussingault, 67 Strasbourg, France).

March 23-25, Use of Small Accelerators for Teaching and Research, Oak Ridge (Mr J. L. Duggan, Special Training Division, Oak Ridge Associated Universities, PO Box 117, Oak Ridge, Tennessee 37830, USA).

March 23-25, Structural Dynamics, Loughborough (Mr $J$. M. Charles, Department of Transport Technology, Loughborough University of Technology, Loughborough, Leicestershire, UK).

April 1-4, Thermodynamics, Cardiff (Meetings Officer, The Institute of Physics and the Physical Society, 47 Belgrave Square, London SW1).

April 6-8, European Congress on Perinatal Medicine, London (Professor P. J. Huntingford, St Mary's Hospital Medical School, London W2).

April 6-10, Analytical Chemistry, Birmingham (Society for Analytical Chemistry, 9-10 Savile Row, London W1). April 7-10, Experimental Stress Analysis, Cambridge (Institution of Mechanical Engineers, 1 Birdcage Walk, London SW1).

April 8-9, Nuclear Gas Turbines, Leicester (Secretary, British Nuclear Energy Society, 1-7 Great George Street, Westminster, London SW1). 